\title{
LES REPAS FUNÉRAIRES: UN TÉMOIGNAGE D'UNE DYNAMIQUE SOCIO-CULTURELLE EN AFRIQUE ROMAINE
}

\author{
Arbia Hilali
}

\begin{abstract}
A la mémoire d'Aelia Secundula. Tous, nous avons déjà assurément payé beaucoup, comme il convient; nous avons en outre décidé d'ajouter à l'autel de notre défunte mère Secundula une table de pierre, sur laquelle rappelant nombre de grandes choses qu'elle a faites, quand les mets auront été apportés les coupes et les couvertures, pour apaiser la blessure cruelle qui ronge en notre cour, tard le soir, nous faisons des récits et louons notre chaste et bonne mère; vieille, elle dormit, celle qui nous a nourris. Tu gis, toujours sobre. Elle a vécu 75 ans. L'an 260 de la province, Iulia Statulenia a fait cela.

(CIL VIII 20277; Satafis, cat. $n^{\circ} 2$ )
\end{abstract}

Les gestes funéraires sont de précieux témoins sur l'organisation des sociétés, leurs croyances, leur économie et sur les liens qui les unissent avec les autres. Si, dans le cadre des repas festifs, le partage de la nourriture renforce les liens entre les convives et le maintien des relations sociales, les repas en l'honneur des défunts unissent vivants et morts dans ce qu'on pourrait appeler une fête alimentaire. L'interprétation des données de fouilles dans une nécropole comme la lecture d'épitaphes offrent à l'historien un champ de réflexion sur ces rites funéraires. Les fouilles récentes dans les nécropoles d'Afrique du Nord, notamment à Pupput en Tunisie, identifient des épitaphes, du mobilier funéraire, des traces d'aliments et des aménagements architecturaux pour les repas funéraires. Célèbre est la mésaventure de Monique, mère d'Augustin, qui, lors de son séjour à Milan, voulut apporter, selon la coutume africaine, "de la bouillie, du pain et du vin pur" aux tombeaux des saints, et fut repoussée par le gardien. ${ }^{1}$ Ces diverses sources nous permettent de collecter et d'analyser cette mémoire en l'inscrivant sur une longue durée. Elles nous invitent à s'interroger sur le contenu de ces cérémonies, les sociabilités exprimées, la dynamique de ces rites et sur la manière

\footnotetext{
${ }^{1}$ Augustinus, Confessiones 6.2.2.
} 
dont le fait de manger et de boire, dans le monde romain, est une manière de croire. ${ }^{2}$

\section{L'espace funéraire, un lieu de commémoration}

La nécropole est un espace de mémoire qui entoure la cité. Notre référence incontournable pour l'Afrique du Nord est la nécropole de Pupput. Depuis la fin des années 1990, la fouille a dégagé près de 2000 tombes sur 7 hectares. Les tombes y sont rassemblées dans des enclos familiaux, qui étaient délimités par des murs assez hauts. Il y avait une gestion collective de l'espace funéraire, par la présence des murs qui préservaient l'intimité des proches lors des cérémonies funéraires et empêchaient aussi l'usurpation des tombeaux. Crémation et inhumation coexistaient au début du IIème siècle. De la crémation individuelle, on passa à la crémation collective, puis c'est l'inhumation qui l'emporta, et les pratiques funéraires s'uniformisèrent au IIIème siècle. ${ }^{3}$ Nous avons d'autres témoignages des pratiques funéraires en Maurétanie à Sitifis, ${ }^{4}$ à Satafis, ${ }^{5}$ à Tipasa ${ }^{6}$ et à Caesarea $;^{7}$ en Numidie, à Thamugadi; ${ }^{8}$ et en Proconsulaire à

\footnotetext{
${ }^{2}$ Pour reprendre l'expression de J. Scheid dans son livre, Quand faire, c'est croire: les rites sacrificiels des Romains (Paris 2005).

${ }^{3}$ A. Ben Abed - M. Bonifay - M. Griesheimer, 'L'amphore maurétanienne de la station $48 \mathrm{du}$ forum des corporations identifiée à Pupput (Hammamet, Tunisie)', Antiquités Africaines 35 (1999), 169-175; A. Ben Abed - M. Griesheimer, 'Fouilles de la nécropole romaine de Pupput (Tunisie)', Comptes Rendus de l'Académie des Inscriptions et Belles-Lettres (2001), 553-590; id., La nécropole romaine de Pupput (Rome 2004) (= Ben Abed - Griesheimer 2004a); id., 'Les supports des offrandes funéraires dans la nécropole de Pupput', dans: M. Fixot (ed.), Paul-Albert Février de l'Antiquité au Moyen-âge. Actes du Colloque, Fréjus 2001 (Aix-en-Provence 2004), 309-324 (= Ben Abed - Griesheimer 2004b); P. Corbier - M. Griesheimer, L'Afrique romaine 146 av. J.-C.- 439 ap. J.-C. (Paris 2005), 297-337; A. Ben Abed - M. Griesheimer, 'Pupput (Hammamet), une nécropole d'époque romaine', Les dossiers d'archéologie 330 (2008), 82-91.

${ }^{4}$ R. Guéry, La nécropole orientale de Sitifis: fouilles de 1966-1967 (Paris 1985).

${ }^{5} \mathrm{P}$-A. Février, 'Remarques sur les inscriptions funéraires datées de Maurétanie Césarienne orientale', Mélanges de l'Ecole Française de Rome 76 (1964), 105-172.

${ }^{6}$ M. Bouchenaki, Les fouilles de la nécropole de Tipasa: 1968-1972 (Alger 1975).

${ }^{7} \mathrm{Ph}$. Leveau, 'Une mensa de la nécropole occidentale de Cherchel', Karthago 18 (1978), 127-131.

${ }^{8}$ P-A. Février, 'Le culte des morts dans les communautés chrétiennes durant le IIIe siècle', dans: Atti del Congresso Internationale di Archeologia Cristiana I, Roma 1975 (Rome 1978), 211-274.
} 
Theveste, ${ }^{9}$ à Thugga $^{10}$ et à Thaenae. ${ }^{11}$ Assez souvent le rite alimentaire se matérialise par une trace monumentale, les mensae qui sont les supports des offrandes funéraires.

En effet, les anciens désignent par ce mot des équipements assez différents dans leurs formes: table ou autel. En Afrique, l'usage épigraphique du mot apparaît sur des épitaphes païennes ou chrétiennes datables de la fin du IIIème siècle. ${ }^{12}$ Les découvertes de la nécropole romaine de Pupput, qui a livré pour l'heure 156 mensae, sont toutes antérieures aux premières attestations épigraphiques de ce mot en Afrique. ${ }^{13}$ Dans cette nécropole, les mensae sont de petits massifs maçonnés. ${ }^{14}$ On remarque la médiocrité de leur construction et l'absence de décors. ${ }^{15}$ En revanche, ailleurs on note un décor tracé ou peint qui rappelle des offrandes ou bien des ustensiles sont sculptés à l'occasion sur de mensae du Sitifis, ${ }^{16}$ du Thamugadi ${ }^{17}$ et $\mathrm{du}$ Theveste. ${ }^{18}$ Cependant le repas funèbre ne fait pas

${ }^{9}$ F. Khadra, 'Nécropole tardive de l'antique Thévèste: mosaïques funéraires et mensae', dans: L'Africa romana VI (Sassari 1989), 265-282.

${ }^{10}$ P. Monceaux, 'L'inscription des martyrs de Dougga et les banquets des martyrs en Afrique', Bulletin Archéologique du Comité des Travaux Historiques et Scientifiques (1908), 87-104.

11 A $10 \mathrm{~km}$ à l'est de Sfax, des découvertes anciennes révélent une importante nécropole présentant des cupules équipées de mensae. Voir Barrier - Benson, 'Fouilles à Thina (Tunisie)', Bulletin Archéologique du Comité des Travaux Historiques et Scientifiques (1908), 22-58. Toutefois, les recherches entreprises ces dernières années sur ce site ne semblent pas avoir livré de nouvelles mensae (N. Jeddi, 'À propos d'une nécropole à Thina, note préliminaire', dans: P. Trousset [ed.], L'Afrique du Nord antique et médiévale: monuments funéraires, institutions autochtones. VIe Colloque International sur l'Histoire et l'Archeologie de l'Afrique du Nord, Pau 1993 (Aix-en-Provence 1995), 139-151.

${ }^{12}$ Ben Abed - Griesheimer 2004b, op.cit. (n. 3), 310.

${ }^{13} \mathrm{Ibid}$. A la fin de l'an 2000 , le pourcentage de tombes équipées de mensae était de $11 \%$ (1393 tombes et 156 mensae).

${ }^{14}$ Ibid. Les dimensions des mensae sont de 50-60 cm de long, une quarantaine de centimètres de large et une hauteur moyenne de $15-20 \mathrm{~cm}$. Ces mensae présentent toutefois une réelle singularité: leur face supérieure n'est pas plane, ni concave en leur centre comme c'est parfois le cas dans d'autres régions africaines.

${ }^{15}$ Ibid. Sur les rivages du golfe d'Hammamet, les mensae ne sont pas décorées, un simple enduit de chaux suffisait. Dans un cas, l'ouvrier a souligné les bords latéraux d'une bande rouge.

${ }^{16}$ Guéry 1985, op.cit. (n. 4), 44, fig. 28.

${ }^{17}$ Février 1978, op.cit. (n. 8).

${ }^{18}$ Khadra 1989, op.cit. (n. 9). A Théveste, dans la nécropole du quartier de Draaer-Rahou, a trouvé une mensa en pierre, ornée en surface de plats et d'ustensiles de cuisine, qui fut réutilisée dans une tombe à coffre tardive. 
systématiquement appel à une monumentalisation. ${ }^{19}$ A côté des traces archéologiques, l'épigraphie révèle la présence de mensae en Maurétanie à Caesarea ${ }^{20}$ et à Satafis. ${ }^{21}$ Les inscriptions funéraires de Maurétanie césarienne orientale évoquent dans des formulations variées le mot mensa: mensa aeterna (cat. $\left.\mathrm{n}^{\circ} 14\right)$, mensa memoriae (cat. $\mathrm{n}^{\circ} 15$ ), mensa perpetua (cat. ${ }^{\circ} 9$ ). On note aussi la diversité des pratiques funéraires. Ainsi, la mensa de la nécropole occidentale de Caesarea, qui pouvait remonter au IIème siècle, ${ }^{22}$ est construite sur une incinération, alors que les mensae connues à Tipasa ont été édifiées sur des sarcophages ou des hypogées à inhumation. ${ }^{23}$ En revanche, dans les mensae de la zone nord et centrale de Pupput, on pratique aussi bien l'incinération que l'inhumation. ${ }^{24}$

\section{Boire et manger, c'est se souvenir de la mémoire du défunt}

La question qui doit, pour le moment, nous retenir est la disposition matérielle de ces repas. Il y a certainement eu dans le monde romain des évolutions: l'une d'entre elles en Afrique a été l'adjonction ou la substitution à la petite table, que l'on plaçait devant la tombe, de véritables lits qui ont matérialisé de façon permanente le repas. Ces lits ont pu, comme on le voit dans la nécropole de Tipasa, être disposées autour d'une dalle de pierre sur laquelle se trouvent l'inscription et le mot mensa; parfois dans ces cas, la dalle a pu servir à déposer le repas. Dans des systèmes plus complexes, le lit est très vaste et un espace vide, au milieu, permet d'imaginer un service plus complexe, sur le modèle du repas d'une maison aisée. ${ }^{25}$ Dans l'ensemble, la nécropole de Tipasa nous a fourni trois formes de

\footnotetext{
${ }^{19}$ P-A. Février, 'Paroles et silences (à propos de l'épigraphie africaine)', dans: L'Africa Romana IV (Sassari 1987), 191.

${ }^{20}$ Leveau 1978, op.cit. (n. 7), 127.

${ }^{21}$ Catalogue $\mathrm{n}^{\circ} 1,2,4,7,9,10,11,12,15,16,17$, 18. J.-M. Lassère, 'Nécropoles, monuments et rites funéraires: période romaine. Bilan épigraphique', dans: Trousset 1995, op.cit. (n. 11), 103-109. Il constate que le terme mensa est très fréquent dans les épitaphes, spécialement en Maurétaine Césarienne et Sitifienne (p. 108).

${ }^{22}$ Leveau 1978, op.cit. (n. 7), 127.

${ }^{23}$ Ibid., 131; P-A. Février, 'Le culte des martyrs en Afrique et ses plus anciens monuments', Corsi di Cultura sull'Arte Ravennate et Bizantina 17 (1970), 191-199. La mensa est construite sur un sarcophage.

${ }^{24}$ Ben Abed - Griesheimer 2004b, op.cit. (n. 3), 313-315.

${ }^{25}$ Février 1978, op.cit. (n. 8).
} 
mensae: la mensa en forme demi-circulaire, la mensa à mosaïque et la mensa à canalisations. ${ }^{26}$ En effet, dans une area de la nécropole occidentale de Tipasa, deux tombes présentent un curieux dispositif avec un petit réservoir et un écoulement possible d'eau, devant l'espace où les personnes appelées au banquet pouvaient s'allonger. ${ }^{27}$ Dans une autre partie de la nécropole, qui avoisine la basilique de l'évêque Alexandre, sont alignés des lits et tables de repas. ${ }^{28}$

Ces lits de repas funéraires de Tipasa sont étroitement liés à un puits ou, parfois même, à une petite citerne qui paraît avoir servi à répandre de l'eau sur la mensa. ${ }^{29}$ Le phénomène n'est pas propre à Tipasa. A Caesarea, sous l'inscription (cat. $\mathrm{n}^{\circ} 1$ ) s'ouvrait l'orifice d'un conduit de libation s'enfonçant de $36 \mathrm{~cm}$ dans le socle. ${ }^{30} \mathrm{~A}$ Pupput, les fouilles ont dégagé des conduits à libation. ${ }^{31} \mathrm{~A}$ Carthage, dans les cimetières des officiales, les tombes sont traversées par un tube ou un conduit, qui permettait d'envoyer au mort sa part de festin. $^{32}$ Au-dessus de la tombe, en avant de la stèle, une table (mensa), généralement rectangulaire, quelquefois demi-circulaire, où étaient représentés, en creux ou en relief, des plats, des patères, des cuillères, des poissons et d'autres mets. ${ }^{33}$ Dans la région de Sitifis, on note la présence d'autels votifs à la fin du IVème siècle. Ces autels étaient connus dans la culture punique et dans les cultes païens de l'époque romaine. Selon N. BENSEDDIK, il s'agit d'une cavité sacrificielle sur le monument. ${ }^{34} \mathrm{~N}$. DUVAL rappelle que les cuvettes creusées dans un cadre quadrangulaire sont présentes dans les églises et les nécropoles aussi bien en Afrique que dans les Balkans. Pour lui, il est peu probable qu'ils servaient à des sacrifices, mais on peut penser aussi à des tables de libation, bien attestées en contexte funéraire jusqu'à la fin du IVème siècle. ${ }^{35}$

${ }^{26}$ Bouchenaki 1975, op.cit. (n. 6), 32, 40-41, 48-49; fig 129-130 (mensa à mosaïque); fig 136 (mensa à canalisations).

${ }^{27}$ Ibid., 50.

${ }^{28}$ Ibid., 52.

${ }^{29}$ Ibid., 87.

${ }^{30}$ Leveau 1978, op.cit. (n. 7), 130.

${ }^{31}$ Ben Abed - Griesheimer 2004b, op.cit. (n. 3), 317.

${ }^{32}$ Monceaux 1908, op.cit (n. 10), 101.

${ }^{33}$ S. Gsell, Les monuments antiques de l'Algérie II (Paris 1901), 47-49; Khadra, op.cit. 1989 (n. 9).

${ }^{34}$ N. Benseddik, 'Autels votifs de la région de Sétif païens et chrétiens', dans: Trousset 1995, op.cit. (n. 11), 185.

${ }^{35}$ Ibid. 
Pour les riches sépultures de famille, les repas se célébraient soit dans le caveau même, soit dans une dépendance de la tombe, dans une salle des banquets (apparitorium, cubiculum, triclinium), qui était aménagée tantôt à côté du caveau, tantôt au-dessus (tombes à étages). On a trouvé en Afrique des sépultures de ce genre, à étage: en bas, la chambre funéraire; au-dessus, une salle pour les banquets. ${ }^{36}$ Quand la sépulture était modeste, le repas se faisait simplement autour de la pierre tombale.

Le rituel alimentaire se passait le soir ${ }^{37}$ et se déroulait par terre ou sur une mensa en maçonnerie. On disposait des mets (un poisson et du pain) qui avaient été apportés dans de petits paniers (sportulae), en même temps que du vin en bouteille, qui était mélangé avec de l'eau chaude avant d'être servi dans des cruches ou des coupes. L'on buvait un mélange de vin et d'eau chaude: c'est la mixsio à laquelle font allusion des textes. ${ }^{38}$ Tel apparaît le convivium auquel la mosaïque de Tipasa invite Paix et Concorde (cat. $\mathrm{n}^{\circ}$ 19). Les fouilles de la nécropole à Pupput offrent des informations supplémentaires sur le rite funèbre adopté pour les défunts dont la sépulture avait reçu une mensa. ${ }^{39}$ La surface de la mensa a souvent été noircie par des traces de fumée qui prouvent que le rituel nécessitait du feu allumé sur la mensa même. Parfois, sous l'effet d'une excessive chaleur, l'enduit éclatait. C'était donc un rituel qui nécessitait la cuisson ou le réchauffement d'aliments, comme le prouve l'abondance des cendres accumulées autour des mensae. ${ }^{40}$

De quoi était composé le menu? Sans doute correspondait-il à ce que consommaient habituellement les vivants, puisque ces derniers devaient partager leur repas avec le disparu. ${ }^{41} \mathrm{~A}$ Caesarea, on a recueilli dans une mensa du matériel tel que lampe, verre et poteries. ${ }^{42}$ Dans ces banquets, les éléments essentiels sont, outre les personnages, boissons, aliments et ustensiles (paniers, amphores,

${ }^{36}$ Gsell 1901, op.cit. (n. 33), 56-99.

${ }^{37} \mathrm{P}$-A. Février, 'La tombe chrétienne et l'au-delà', dans: Le temps chrétien de la fin de l'Antiquité au Moyen Age, III-XIII siècles, Paris 1981 (Paris 1984), 165. Le texte de Satafis (cat. $\mathrm{n}^{\circ}$ 2) montre que la cérémonie s'est déroulée tard le soir.

${ }^{38}$ CIL IV 1292a.

${ }^{39}$ Ben Abed - Griesheimer 2004b, op.cit. (n. 3), 313-315.

${ }^{40}$ Ibid., 317.

${ }^{41}$ L. Bachelot, 'Nourrir les morts', in B. Lion, Banquets et fêtes au procheOrient ancien: les banquets des dieux, les festins des rois, la réception de luxe, régimes alimentaires (Dijon 2003), 84.

${ }^{42}$ Leveau 1978, op.cit. (n. 7), planches 28-29. 
cruches ou gobelets). Le poisson apparaît sur les tables d'offrandes funéraires qui accompagnent des tombes non chrétiennes et aussi dans des régions où sa consommation ne pouvait être que très réduite, ainsi à Thamugadi. ${ }^{43}$ Les fouilles mettent aussi en évidence un rituel de libation dont témoignent les patères retrouvées par dizaines autour des mensae $e^{44}$ ainsi que les mensae à canalisation à Tipasa, ${ }^{45}$ à Caesarea ${ }^{46}$ et à Pupput. ${ }^{47}$ Lors de la construction de certains mausolées, il était parfois prévu d'annexer au monument un vignoble dont le produit serait spécialement destiné aux libations funéraires. Ainsi, aux bords du désert saharien, les Flavii de Cillium ont réservé une parcelle de vigne dans le jardin entourant leur mausolée. ${ }^{48}$ Ces repas peuvent générer des excès de beuverie et Augustin reconnaît son impuissance à les faire cesser, même sur les tombes de martyrs. ${ }^{49}$ Il critique le sens que les contemporains donnent au repas: "aux yeux du peuple charnel et ignorant, ces beuveries et ripailles dans les cimetières" peuvent faire croire "non seulement qu'ils honorent les martyrs, mais soulagent les morts". ${ }^{50} \mathrm{Il}$ souligne avec ironie que les hommes qui font de tels repas "rendent ainsi service à leur ventre et non aux âmes de leurs morts" 51 et qu'on ne fait ainsi que reprendre une tradition païenne, les parentalia.

\section{Le repas funèbre, un geste rituel fonctionnel}

Le repas funèbre a une fonction juridique qui a pour finalité l'intégration de la famille du défunt dans la vie de la cité. Pendant la période du deuil, les parents du défunt se trouvaient impliqués dans le contexte de la mort et exclus de la vie sociale de la cité. En effet,

\footnotetext{
${ }^{43}$ Février 1978, op.cit. (n. 8), 251.

${ }^{44}$ Ben Abed - Griesheimer 2004b, op.cit. (n. 3), 317.

${ }^{45}$ Bouchenaki 1975, op.cit. (n. 6), 32, 40-41, 48-49.

${ }^{46}$ Ibid., 48-49; Leveau 1978, op.cit. (n. 7), 130.

${ }^{47}$ Ben Abed - Griesheimer 2004b, op.cit. (n. 3), 317.

${ }^{48}$ CIL VIII 211-216; J.-M. Lassère, Les Flavii de Cillium (Paris 1993); cf. aussi Petronius, Satyrica, 71, 7: "Je veux qu'il y ait toutes sortes de fruits autour de mes cendres et des vignes en abondance".

${ }_{50}^{49}$ Augustinus, De civitate Dei 8.27.

50 Augustinus, Epistulae 22.6. Cf. Tertullianus, Apologeticum 39.17-19: il évoque la tradition de repas entre les chrétiens sans excès, précédé par une prière à Dieu.

${ }^{51}$ Augustinus, Sermones 361.6.

${ }^{52}$ Augustinus, Confessiones 6.2.
} 
le défunt et sa famille étaient impliqués dans l'espace de la mort. Les parents du défunt étaient coupés de leur environnement normal, notamment des temples, des magistrats et prêtres et des divinités d'en haut. ${ }^{53}$ Ce qui explique que la cérémonie se déroulait la nuit, discrètement, en évitant tout contact visuel avec les temples et les lieux publics. Le partage sacrificiel effectué auprès de la tombe purifie la famille du contact avec la mort, c'est-à-dire la réinsérait dans la société des vivants. En même temps le défunt entrait définitivement dans sa nouvelle demeure et acquérait son nouveau statut. Une fois, la tombe fermée, le défunt rejoignait progressivement les dieux Mânes, et la famille endeuillée retrouvait peu à peu sa place parmi les vivants. La famille "souillée par la mort" (funestata) était progressivement purifiée (purgata), ce qui signifie que, d'un point de vue juridique, elle retrouvait son identité et sa capacité juridique, perdues au moment du décès de son proche. La personne défunte recevait également de nouveaux droits: celui, notamment, d'être protégée dans sa tombe par la cité et d'être régulièrement honorée. Ces honneurs la reconnaissaient comme un membre mort de la famille et de la cité et comme une partie de la divinité collective des Mânes. ${ }^{54}$

Cette séparation entre le monde des morts et celui des vivants se traduit dans l'espace funéraire par le fait que la vaisselle du défunt était symboliquement détruite à la suite d'un repas funèbre. Dans la nécropole de Pupput, malgré le grand nombre de patères récoltées, aucune n'a été retrouvée intacte, toutes ont été brisées, parfois même simplement retournées puis écrasées sur le sol où leurs morceaux dessinent encore une forme complète. On a trouvé dans une tombe, un vase en sigillé, retourné et volontairement brisé, trace d'un geste rituel. ${ }^{55}$ On note aussi l'aspect très coloré des tombes avec des fleurs qui marquent la limite entre le monde vivant coloré et l'au-delà sombre, marécageux. C'est un message adressé aux vivants pour inviter à profiter pleinement de la vie. Sur le centre de une mensa de Satafis (cat. $\mathrm{n}^{\circ} 3$ ), est dessinée une rosace à six branches. Sur une autre mensa du même lieu sont représentées des guirlandes et des colombes (cat. $\left.n^{\circ} 7\right) .{ }^{56}$ E. BERNAND fait remarquer, à propos des

\footnotetext{
${ }^{53}$ Scheid 2005, op.cit. (n. 2), 184-185.

${ }^{54}$ Ibid., 187.

${ }^{55}$ Ben Abed - Griesheimer 2004b, op.cit. (n. 3), 317.

${ }^{56}$ Février 1964, op.cit. (n. 5), 133.
} 
inscriptions métriques d'Egypte, que ce ne sont pas seulement les survivants qui pleurent sur les morts; mais aussi les défunts qui formulent des souhaits pour les vivants: En particulier on relèvera: "Puissiez-vous demeurer perpétuellement sur la terre, aussi longtemps que j'habiterai la maison de Perséphone". ${ }^{57}$

Ce rituel alimentaire assurait la paix du défunt dans sa nouvelle demeure. L'organisation du monde voulue par les dieux pouvait être menacée par les morts qui n'avaient pas reçu de demeure, de tombe et que l'on n'avait donc pas pu nourrir: les fantômes errants présentaient à cet égard un immense danger. La terreur des revenants hantait les imaginations. ${ }^{58}$ Les Anciens croyaient que les morts avaient ont faim et soif et que, si l'on ne satisfaisaient pas leurs besoins, ils ne pouvaient pas vivre en repos. ${ }^{59} \mathrm{La}$ nourriture, réellement ou symboliquement consommée avec eux, assurait, en paix, une continuité indispensable entre le monde des morts et celui des vivants. ${ }^{60}$ Cette quête de tranquillité après la mort se manifeste dans les inscriptions par des formules comme hic requiescit, ossa quiescant, sit sibi terra levis. Sur une épitaphe, le dédicant se félicite qu' "après une lourde charge et d'incessants labeurs, il se tait maintenant, et se satisfait de ce séjour silencieux et se repose". ${ }^{61} \mathrm{La}$ même atmosphère de paix, de concorde, de vie heureuse autour d'une bonne table, parmi des compagnons joyeux, que l'on souhaitait tant aux morts qu'aux vivants, se retrouve dans les monuments funéraires chrétiens. ${ }^{62}$ Elle caractérise aussi bien les scènes de banquets des peintures des catacombes ou des sarcophages sculptés de Rome que des textes funéraires d'Afrique, comme cette phrase, si significative, inscrite sur la mosaïque d'une mensa du cimetière de

${ }^{57}$ E. Bernand, Inscriptions métriques de l'Egypte gréco-romaine (Paris 1969), 27.

${ }^{58}$ Février 1978, op.cit. (n. 8), 211-274.

${ }^{59}$ F. Cumont, Recherches sur le symbolisme funéraire des Romains (Paris 1942), 354.

${ }^{60}$ Bachelot 2003, op.cit (n. 41), 86.

${ }^{61}$ CIL VIII 5278: Qui post tantum onus, multos crebosque labores, nunc silet et tacito contentus sede quiescit; Apuleius, Metamorphoses 2.29: un mort ranimé par un magicien, se plaint d'avoir été dérangé par lui.

${ }^{62}$ Monceaux 1908, op.cit (n. 10), 96 : „Les banquets funéraires et les agapes étaient si populaires, que, par une curieuse transposition où se trahissent les aspirations réalistes des foules, on en faisait l'une des joies du Paradis." 
Tipasa: in Christo Deo pax et concordia sit convivio nostro (cat. $\mathrm{n}^{\circ}$ 19). ${ }^{63}$

Le banquet funéraire constitue un rite de sociabilité important. ${ }^{64}$ Il conserve et pérennise la mémoire collective. En effet, l'un des liens essentiels qui maintenait dans une même communauté ceux qui avaient quitté ce monde et ceux qui s'y trouvaient encore était la nourriture. La mort ne signifie nullement le néant et un défunt devait être régulièrement nourri, même si son existence se poursuivait désormais dans un monde souterrain. ${ }^{65}$ L'anthropologie a depuis longtemps établi l'identité fonctionnelle du voir et du manger, de la perception visuelle et de l'absorption effective d'aliments. La présence des images évoque le repas funèbre (la sculpture sur les sarcophages ou la mosaïque), la poterie déposée auprès du mort rappelle ce rituel lointain. ${ }^{66}$ Ainsi l'alimentation des morts avait-elle la fonction essentielle de maintenir la pérennité du groupe social, malgré la disparition des individus. Certaines inscriptions expriment ce souhait en gravant sur la pierre mensa aeterna ou perpetua $\left(\right.$ cat. $\mathrm{n}^{\circ}$ 14 et 9). Le repas sur la tombe est l'occasion d'assurer la stabilité et l'unité de la famille. A Satafis, une conversation s'est engagée entre les enfants à propos leur mère (cat. $\mathrm{n}^{\circ} 2$ ).

La mort antique, par sa présence aux portes mêmes de la ville, de part et d'autre des voies principales, n'a jamais cessé de réunir à des dates répétées et la famille et l'ensemble de la société. ${ }^{67}$ Cette mort n'est pas écartée; elle est insérée dans le jeu des relations sociales et du vécu. Elle emprunte les formes de ses célébrations aux gestes qui assuraient la force de la structure sociale. Comme l'écrit Valère Maxime, "nos ancêtres instituèrent un repas annuel, nommé caristia, où l'on n'admettait que les parents et les alliés, afin que, s'il existait quelque différent dans la famille, des esprits pacifiques intervenaient

${ }^{63}$ L'expression in Deo est très fréquente dans les inscriptions chrétiennes. Elle fait partie des acclamations prononcées par les parents du défunt. Quant au mot pax, il se retrouve dans plusieurs épitaphes sépulcrales: CIL VIII 6957, 9751, 9752bis, 9793, 9815, 21635, 21637, 21642-43, 21645, 23920.

${ }^{64}$ Scheid 2005, op.cit. (n. 2), 167.

${ }^{65}$ Bachelot 2003, op.cit (n. 41), 83.

${ }^{66}$ Ibid., 85.

${ }^{67}$ Scheid 2005, op.cit. (n. 2), 167-179: cette forme du culte des morts pouvait être célébrée le jour de l'enterrement (silicernium), au neuvième jour après la mort (novemdialis), pour l'anniversaire (dies natalis du jour de la naissante chez les païens, de la mort chez les chrétiens, et surtout le 22 Février (caristia, cara cognatio), afin de clôturer une semaine consacrée aux Mânes (parentalia). 
pour y mettre fin, de rétablir la bonne intelligence entre les proches" ${ }^{68}$ La tombe demeure donc, comme la maison, le lieu où se dévoilent les différences, comme aussi les gestes répétitifs qui garantissent l'ordre. De nos jours, on peut se demander comment sont perçus ces tombeaux antiques en Afrique du Nord. Le souvenir de ces morts lointains n'a pas totalement disparu de l'espace mental actuel. Dans le langage de certains villageois en Tunisie, on conserve le toponyme "masoula" ou "msoula" appliqué à des mausolées ou, plus souvent, à des tumulus. L'archéologue N. FERCHIOU a rapproché ce terme de mausoleum. Selon cette hypothèse, on est en présence d'un mot latin conservé dans le parlé local. Cette permanence dépasse dans la mémoire collective le cadre linguistique et persiste dans une pratique funéraire. En effet, les tumulus font souvent l'objet d'un culte populaire des saints ("mzara"). ${ }^{69}$

Étudier les gestes autour de la mort et les étudier sur la longue durée, afin d'en noter les continuités et les ruptures, révèle autant le dynamisme d'une société que les processus d'acculturation qui l'animent, entre Africains, Romains et Africains romanisés. Nos sources permettent d'évaluer les progrès de la romanité chez les Africains et cette adaptation aux différents rites d'incinération et d'inhumation. Ces banquets funéraires ont survécu et ont pris une grande importance dans l'antiquité tardive et furent christianisés et plus tard islamisés. $^{70}$

Les repas funéraires nous permettent de comprendre les rapports de l'individu, mais aussi de la communauté, avec la mort, pour clarifier la stratification mentale complexe qui s'est élaborée, et pour reconstruire les comportements. Les étudier permet de réhabiliter les gestes et leur fonction et d'établir une équation entre la croyance et

${ }^{68}$ Valerius Maximus, Facta et dicta memorabilia 2.8 (ed. P. Constans [Paris 1935], 104-105).

${ }^{69}$ N. Ferchiou, 'Architecture funéraire de Tunisie', dans: Trousset 1995, op.cit. (n. 11), 137.

${ }^{70}$ Augustinus, Confessiones 6.2. Il critique cette pratique païenne puisqu'il compare les repas funèbres des chrétiens aux parentalia des païens. A Milan, Saint Ambroise donne l'exemple en interdisant les repas funèbres dans les cimetières. Cf. Augustinus, Contra Faustum 20, 21. Cf. C. Lepelley, 'Formes païennes de sociabilité en Afrique au temps de Saint Augustin', dans: F. Thélamon (ed.), Sociabilité, pouvoirs et société. Actes du Colloque, Rouen 1983 (Rouen 1987), 99103. En partant d'une expérience personnelle, lors de mes visites à la mémoire de mes grands parents au cimetière à Tunis, j'ai pu observé des familles en train de manger et boire avec joie autour de la tombe du défunt. 
l'action. La pratique des rites funéraires exprime un des aspects de la religion romaine, une religion communautaire, ritualiste et fonctionnelle. En effet, tant que la famille ou la communauté célébrait les banquets, la personne décédée survivait en tant que membre de cette famille. Si les rites funéraires n'étaient pas célébrés, le défunt sombrait définitivement dans la non-existence. Ces rites montrent, en définitive, que, chez les Romains, la survie des morts ne dépendait pas d'une volonté divine mais d'une communauté humaine et de ses rites. ${ }^{71}$ Malgré l'abondance de l'épigraphie funéraire en Afrique du Nord, on remarque qu'il y a peu de trace épigraphique pour mémoriser le rituel du repas funèbre. Les parents n'utilisent pas souvent l'écrit pour pérenniser la mémoire. Cette dernière se conserve autrement, par le geste des survivants de la famille qui reviennent accomplir leur rite alimentaire sur et autour de la tombe. Il va sans dire qu'une partie du dossier nous échappe et, singulièrement, la possibilité d'avoir une vision globale d'un contexte funéraire où seraient étudiées les relations entre sépultures, supports d'offrandes et offrandes. Il est difficile et toujours dangereux de faire parler les morts, mais qui pourrait mieux qu'eux parler de ce qu'ils étaient et de ce qu'ils faisaient dans leurs pratiques rituelles.

Nanterre, Décembre 2007

${ }^{71}$ Scheid 2005, op.cit. (n. 2), 188. 


\section{Catalogue}

1) P. Leveau, 'Une mensa de la nécropole occidentale de Cherchel', Karthago 18 (1978), 129-130 = AE 1978, 896 (Caesarea Mauretaniae; $\mathrm{II}^{\mathrm{e}}$ siècle)

Marcia Roga[ta] / Cyt[i]sis annor(um) XV, [men]s(ium) [---]. / $H[a e c$ f] uit pia et sapiens / ultramodum aetatis, velut / contendente sensu cum / celeritate fatorum.

"Marcia Rogata Cytisis, 15 ans, [---] mois; elle a vécu ici pieuse et sage, plus qu'il n'est de son âge, comme si elle luttait par l'esprit avec la rapidité des destins".

Sous l'inscription s'ouvrait l'orifice d'un conduit de libation s'enfonçant de $36 \mathrm{~cm}$ dans le socle.

2) CIL VIII 20277: tablette (Satafis; a. 299)

Memoriae Aeliae Secundulae. / Funeri mu<l=I>ta quid(e)m condigna iam misimus omnes, / insuper ar(a)equ(e) deposit(a)e Secundulae matri. / Lapideam placuit nobis atponere mensam, / in qua magna eius memorantes plurima facta, / dum cibi ponuntur calicesq(ue) $e<t=I>$ copertae, / vulnus ut sanetur nos rod(ens) pectore saevum, / libenter fabul(as) dum sera red(d)imus hora / castae matri bonae laudesq(ue), vetula dormit; / ipsa [q(uae)] nutri(i)t, iaces, et sobria\{e\} semper. / V(ixit) a(nnis) LXXV. A(nno) p(rouinciae) CCLX. Statulenia Iulia fe/cit.

3) P-A. Février, 'Remarques sur les inscriptions funéraires datées de Maurétanie Césarienne orientale', Mélanges de l'Ecole Française de Rome 76 (1964), $155=A E$ 1972, 770: plaque avec au centre une rosace (Région de Sétif, Novar... / Beni Fuda ; a. 315)

Mensa Kamili / Donati, v(ixit) a(nnis) LXXXXI, / deces(s)it idus Maias / a(nno) p(rovinciae) CCLXXVI. / Fecerunt filii.

4) Ibid., 155: petite dalle carrée (Satafis; a. 329)

Me(n)sa / Iuli/ Ma/sceli, / vi(xit) an(nis) LXI, an(no) p(rovinciae) CCXC. 
5) CIL VIII 20474: dalle carrée (Région de Sétif, Novar... / Beni Fuda; a. 331)

Cerelius Metumus / filius $d<e=I>\operatorname{dik}(a) v i\{v\}(!) /$ mensa patris / mei. Maxim/us v(ixit) an(nis) LXXX, / VII kalendas Apri(les) a(nno) / p(rouinciae) CCXCII.

6) Février 1964, op.cit. ( $\left.{ }^{\circ} 3\right), 156=A E$ 1972, 734: dalle rectangulaire (Sitifis; a. 334)

Mensa Sabassane, vics(it) / annum unu(m), men(ses) tres, / dies XVII. Parentes / amanti posuerunt, (anno) p(rouinciae) CCXCV.

7) CIL VIII 20472 (Satafis; a. 342)

Mensa P(ubli) Aeli / Nampuli, v(ixit) a(nnis) LXXX/III, m(ensibus) IIII. D(e)d(icata) / idus Maias / an(no) p(rovinciae) CCCIII. / Fecit P(ublius) Ael(ius) Donatus filius eius.

8) $A E$ 1903, 111 (Koudiat Adjala; a. 344)

[M]e(n)sa Pauli, vixit annis [---] / [--- qui]evit die VIII kal(endas) Decembr[es] / [a(nno) p(rovinciae)] CCCV vivente Optata co[ninge].

9) CIL VIII 20304: dalle rectangulaire (Satafis; a. 349)

Flor(a)e bon(ae)e m/emori(a)e con/iugi Quetus / maritus mensam / perpetuam posu/it, quae viscit(!) an/nis LX, decessit o/ctav(as?) kal(endas) Martias / anno provi[n]ciae / CCCX.

10) CIL VIII 20303: plaque presque carrée (Satafis; a. 352)

Mensa Aemili/ae Valentin(a)e bene mer/itae de Claudio S/aposo marito suo, / fabente(!) Deo sine dol/ore filioru(m) discessit. Vicxit(!) I an(nis) $L X,<a=M>$ (nno) $p$ (rovinciae) CCCXIII.

11) CIL VIII 20473 (Région de Sétif, Novar... / Beni Fuda; a. 360)

Mensa / haec est aeterna / domus et perpetua / felicitas / de omnibus meis / hoc solum meum. Aper fidelis / in pace vixit an(n)is LXV./ 
Dep(ositio) eius $X k$ (a)l(endes) Sep(tembres) an(no) p(rovinciae) CCCXXI.

12) P. Massiera, 'Note sur des inscriptions chrétienne de Périgotville', Bulletin Archéologique du Comité des Travaux Historiques et Scientifiques (1950), 47, $\mathrm{n}^{\circ}$ 4: dalle carrée (Satafis; a. 362)

Memoria / Caelia Musa / Iacina h(ic) s(ita) e(st). / Mensa posue/runt maritus et fili(i) eius. Vi(xit) an(nis) XL. An(no) p(rouinciae) CCXXIII.

13) Février 1964, op.cit. ( $\mathrm{n}^{\circ}$ 3), 160: dalle rectangulaire (Koudiat Adjala; à partir de 369)

Me(n)sa Barsei, / vixit an(n)is XXII, / an(n)o provinci(a)e CCC/XXX[---].

14) CIL VIII 20478 (Région de Sétif, Novar... / Beni Fuda; a. 374?)

Mensa aeterna / Ianuari, v(ixit) a(nnis) LXXV, / dec(essit) kal(endas) Sept(embres) et m[---] / CXII. H(a)ec est pau[s]a, / h(a)e(c) est d(o)m(us) (a)eterna. / L(ucius) T(ullius) Cicero ei f(ecit) a(nno) p(rovinciae) $C C[C] X X X V$.

15) P. Massiéra, 'Inscriptions chrétienne de Maurétanie sitifienne', Revue Africaine (1956), 326, $\mathrm{n}^{\circ}$ 21: Pierre, (Satafis; a. 389?), inscription revue en 1962.

Mensa memori/ae Iuliae Vale/riae, vicx/it(!) annis t[r]/iginta, disc[es]/it die nonu $k[(a)$ lendas] no]bebbrias a[n(no) p(rovinciae) CCCL ].

16) Ibid., 326, $n^{\circ}$ 17: dalle carrée (Satafis; a. 392)

Mensa Iu/liani, vixit / annis tribus, / depositio IIII n(onas) / Sep(tembres) an(no) p(rovinciae) CCCLIII.

17) Massiéra 1950, op.cit. ( $\left.\mathrm{n}^{\circ} 12\right), 49, \mathrm{n}^{\circ}$ 9: dalle rectangulaire (Satafis; a. 400) 
Me(n)sa Maris Amar/daci qui et Muna/tiani, vixit annis / XXXVI et decessit / XVI kal(endas) Novembres / an(no) p(rovinciae) CCCLX et primo.

18) Ibid., 51, $\mathrm{n}^{\circ}$ 13: dalle rectangulaire. (Satafis; a. 420)

Mensa mem[oriae ---] / Iulia Feli[---] / mansit ann[is---], / discessit $X I$ kale(ndas) De[cembres] / an(no) p(rovinciae) CCCLXII, ABDOVESEM [---] / NIUSCNPASSUS maritus [---] / discessit an(nis) LXXXV, VIII kal(endas) Aprile / an(no) p(rovinciae) CCCLXXXI.

19) J.-M. Blas de Roblès - C. Sintès, Sites et monuments antiques de l'Algérie (Aix-en-Provence 2003), 71: sur une mosaïque (Musée de Tipasa; IV ${ }^{\mathrm{e}}$ siècle).

In Christo Deo pax et concordia sit convivio nostro.

20) Monceaux 1908, op. cit. (n. 10), $90=C I L$ VIII 27333, pierre, (Thugga; fin $\mathrm{IV}^{\mathrm{e}}$ - début $\mathrm{V}^{\mathrm{e}}$ siècle).

Sancti ac b\{a\}eatissimi martyres / petimus in mente habeatis, ut do/nentur vobis [---] simposium, / Mammari(um), Graniu(m), Elpidefo/rum, qui haec cub(icula) quattuor ad c(onvivia) p(ro) m(artyribus?) / suis sum(p)tibus et suis operibus / perfecerunt.

"Saints et bienheureux martyrs, nous vous demandons, pour qu'on vous offre un banquet, de vous souvenir de Mammarius, de Granius, d'Elpideforus, qui ont construit entièrement ces quatre cubicula pour les banquets des martyrs, à leurs frais et avec œuvres par leurs soins". 